\title{
Le Bureau international du travail et la
} modernisation économique dans les années 1920 : esquisse d'une dynamique institutionnelle

ILO and the economic modernization in the 20s : a first study of institutional dynamics

\section{Thomas Cayet}

\section{OpenEdition}

Journals

Édition électronique

URL : http://journals.openedition.org/travailemploi/4561

DOI : 10.4000/travailemploi.4561

ISSN : 1775-416X

Éditeur

DARES - Ministère du Travail

Édition imprimée

Date de publication : 30 mars 2007

Pagination : 15-25

ISSN : 0224-4365

Référence électronique

Thomas Cayet, « Le Bureau international du travail et la modernisation économique dans les années 1920 : esquisse d'une dynamique institutionnelle », Travail et Emploi [En ligne], 110 | Avril-Juin 2007, mis en ligne le 15 juin 2009, consulté le 01 mai 2019. URL : http://journals.openedition.org/ travailemploi/4561 ; DOI : 10.4000/travailemploi.4561 


\title{
Le Bureau international du travail et la modernisation économique dans les années 1920: esquisse d'une dynamique institutionnelle
}

\author{
Thomas Cayet $\left(^{*}\right)$
}

\begin{abstract}
À travers la question de «la modernisation économique » dans l'entre-deux-guerres et l'apparition du Bureau international du travail, l'auteur s'intéresse à l'évolution globale de la question sociale et son intégration avec l'économique. Il vient enrichir l'histoire du rôle des organismes internationaux dans la construction de la société et de l'économie. Il montre que, dès sa création, le Bureau international $d u$ travail lutte pour une intégration de la dimension sociale et économique et décrit les difficultés qu'il rencontre à faire accepter et valoir sa compétence économique. Ceci semble encore pertinent aujourd'hui et apparaît comme une constante du positionnement du Bureau international du travail parmi les organisations internationales.
\end{abstract}

De l'horreur de la guerre doit naître un monde où l'organisation de la justice sociale permet une paix durable. Cet idéal est le fondement de l'Organisation internationale du travail (OIT). Le premier directeur du Bureau international du travail (BIT), Albert Thomas, et le président de son conseil d'administration, Arthur Fontaine, ont été marqués par la création $\mathrm{du}$ ministère du Travail français et leur collaboration à la mobilisation industrielle française. Mais ils perpétuent surtout à Genève l'expérience d'un milieu international de réformateurs. Le BIT entretient un réseau d'influence et un réservoir d'expertise qui lui permettent de faire bouger les lignes des conflits de compétences. Afin de comprendre ce jeu des possibles, il faut analyser son développement institutionnel en essayant de saisir cette dialectique du dedans et du dehors dessinant un espace d'action propre. L'étude du traitement des questions de modernisation économique éclaire ici de manière décalée mais fructueuse les continuités, méandres et contradictions de cet engagement international.

\section{Les fondements d'un développement original}

Le 25 janvier 1919, la Conférence de paix nomme une commission chargée d'étudier la législation internationale du travail. Face à la tentation révolutionnaire, les gouvernements des nations victorieuses mettent en avant l'organisation internationale d'une coopération sociale.

(*) Docteur en histoire de l'Institut universitaire européen. tcayet@iue.it

\section{Créer un «Parlement du travail »}

Samuel Gompers, président de l'American Federation of Labour (AFL), dirige ses travaux. Arthur Fontaine, délégué par le gouvernement français, en est le secrétaire général. Sont également membres les Belges Ernest Mahaim et Emile Vandervelde, les Britanniques Harold Butler et Sir Malcolm Delevingne, les Italiens Angiolo Cabrini et Guglielmo Emanuel Di Palma Castiglione, le Polonais François Sokal ou encore le Tchèque Edouard Benes. Une des caractéristiques de l'Organisation internationale du travail est la continuité revendiquée entre le milieu réformateur d'avantguerre, l'action de ses fondateurs et son fonctionnement ultérieur. Nombre de ces personnalités deviendront des membres du conseil d'administration du BIT ou même entreront à son service (Butler, Cabrini, Di Palma Castiglione) (1).

Les représentants français, Arthur Fontaine, Pierre Colliard, ministre du Travail, et Louis Loucheur, ministre de la Reconstruction industrielle, fondent leurs réflexions à propos de la nouvelle institution sur un rapport rédigé par la Commission du travail de la chambre des députés. Ce texte met en exergue le développement et le dépassement nécessaire des initiatives portées par les associations de réforme sociale. Il esquisse un cadre institutionnel organisé autour de la réunion de grandes conférences définissant une véritable législation internationale du travail. Justin Godard, le concepteur

(1) Pour une bonne synthèse des positions des dirigeants syndicaux, patronaux et gouvernementaux, $c f$. GuÉRIN (1996) et DE Felice (1988). Pour une description des origines du BIT, les travaux et témoignages rassemblés par un des acteurs restent une référence obligée, $c f$. SHOTwELL (1934). 
de ce rapporteur fondateur, deviendra lui aussi une personnalité importante au sein du BIT. En tant que délégué français à son conseil d'administration dans les années 1920 et 1930, il mettra son expérience d'ancien ministre du Travail au service de nombreuses commissions spécialisées, notamment celles des migrations, du chômage ou encore des loisirs. Il présidera même deux fois la Conférence internationale du travail, en 1934 à Genève et en 1948 à San Francisco (2).

La conciliation du respect des souverainetés nationales avec une forme de pouvoir «législatif» à attribuer au nouvel organisme est alors le sujet majeur de deux mois de discussions passionnées. Les propositions émanant du BIT soumises au vote des représentants nationaux lors des Conférences internationales du travail (CIT) annuelles prendront finalement la forme de conventions ou de simples recommandations. Les membres auront obligation de soumettre aux autorités compétentes, dans un délai d'un an à partir de la clôture de la session de la conférence, les conventions ayant obtenu les deux tiers des voix. Ce dispositif repousse toute ingérence directe dans les législations nationales, mais impose juridiquement un débat parlementaire aux États. L'autre question cruciale porte sur la composition des représentations nationales lors des CIT. Une formule tripartite avec double représentation des États est adoptée: deux représentants gouvernementaux, un patronal et un ouvrier. Chaque délégué est censé voter individuellement et non en fonction de son appartenance nationale. Cette intronisation internationale du tripartisme fait de la nouvelle institution un lieu privilégié pour les rencontres transnationales au sein du système de la Société des Nations (SDN) (3).

La partie XIII du traité de Versailles faisant office de constitution pour l'OIT est acceptée par les membres de la Conférence de paix le 11 avril 1919. Lors de la première CIT organisée à Washington en novembre 1919, six conventions sont votées. Elles portent sur la durée journalière et hebdomadaire du travail, avec la convention fondamentale sur les 8 heures, les statistiques du chômage, la protection de la maternité, le travail de nuit des femmes, l'âge minimum du travail et le travail de nuit des enfants. La première session du conseil d'administration du BIT suit immédiatement la conférence de Washington. La nomination de Sir Éric Drummond à la tête du secrétariat de la SDN et le retrait américain avantagent une candidature française. Arthur Fontaine, mandaté par le gouvernement français, et

(2) Pour des précisions biographiques sur Justin Godart, cf. WiEviorka (sous la direction de) (2004) et notamment l'article d'I. Moret-Lespinet sur Justin Godart et le Bureau international du travail.

(3) Concernant l'innovation juridique que constitue cette représentation internationale des intérêts, $c f$. TORTORA (1980) et cf. GHÉBALi (1987).
Léon Jouhaux pour les syndicats, proposent le nom d'Albert Thomas. Robert Pinot convainc également les patrons de soutenir une telle candidature. L'union des représentants français permet sa désignation comme directeur du BIT. Arthur Fontaine est, lors de la même séance, élu président du conseil d'administration (4).

L'organisation de cette structure permanente reprend le mode d'action - la signature de conventions internationales concernant les dispositifs de protection sociale - et l'héritage des associations de droit social qui se sont développées tant au niveau national qu'international à partir de la fin du $\mathrm{XIX}^{\mathrm{e}}$ siècle. Les désignations d'Arthur Fontaine et d'Albert Thomas soulignent le lien fort entre cette nouvelle institution et cette "nébuleuse réformatrice» (5).

\section{Une connaissance de la «question sociale " mise au service du Bureau international du travail}

André Fontaine, polytechnicien du corps des Mines, est entré à l'Office du travail en 1891 et en est le directeur depuis 1899. D'abord rattaché au ministère du Commerce puis, après 1906, au ministère du Travail, l'Office du travail est alors un lieu unique d'études et de rencontres autour des conditions de travail. Arthur Fontaine a participé à la création de l'Association internationale pour la protection légale des travailleurs et, en 1906, à l'élaboration des premières conventions internationales de protection du travail (6).

Albert Thomas a été l'un des grands organisateurs de la mobilisation industrielle française. En décembre 1916, il devient ministre de l'Armement et des Fabrications de guerre. Albert Thomas participe au début du siècle à l'élaboration d'un "socialisme normalien»(7). Ces intellectuels influencés par Émile Durkheim cherchent à définir une culture socialiste nouvelle fondée sur une analyse dite scientifique de la société, en rupture avec le lyrisme politique de la fin du XIX ${ }^{\mathrm{e}}$ siècle. Rédacteur en chef de la Revue socialiste après sa fusion avec la Revue syndicaliste et coopérative en 1910, Albert Thomas, élu député la même année, joue un rôle central au sein de ces réseaux en assurant un lien entre des personnalités plus ou moins bien intégrées dans le mouvement socialiste et les milieux syndicaux. (8) Il collabore à une revue animée par son ami Edgar Milhaud, Les Annales de la régie directe,

(4) Concernant le rôle de Robert Pinot, $c f$. Waline (1957).

(5) Pour l'utilisation de cette expression et l'analyse d'un «champ réformateur», $c f$. C. Topalov (1999).

(6) Concernant Arthur Fontaine et l'Office du Travail, $c f$. I. Lespinet-Moret (2003) et LuCiani (1992).

(7) Pour des précisions biographiques, $c f$. SCHAPER (1960).

(8) Participaient à ces réseaux des proches d'Albert Thomas comme François Simiand, Maurice Halbwachs ou encore Edgar Milhaud. $C f$. Prochasson (1999) et Charle (1994). 
et se familiarise avec les expériences du socialisme municipal et du mouvement coopératif. Membre de la Commission du budget à la chambre des députés, il envisage la possibilité des réformes sociales à travers un dialogue avec des dirigeants patronaux. Lors de la mobilisation industrielle, Albert Thomas travaille avec de nombreuses personnalités du milieu réformateur et notamment Arthur Fontaine. Après son départ du ministère de l'Armement, il préside les réunions du Comité permanent pour l'étude et la prévention du chômage institué par le ministère du Travail pour envisager les perspectives économiques de l'après-guerre.

Ces expériences communes permettent une complicité particulière entre le directeur du BIT et le président de son conseil d'administration. Les bons rapports entre Léon Jouhaux, représentant la CGT, et Albert Thomas font du lien entre ces personnalités un point d'appui important pour le développement de la nouvelle institution. Les contemporains ont souvent glosé sur le partage d'influence entre la Grande-Bretagne et la France concernant le BIT et le secrétariat général de la Société des Nations. Les nominations d'Albert Thomas et de Sir Éric Drummond furent évidemment âprement négociées au niveau diplomatique mais, s'il y a une influence française, elle se fonde surtout sur une connaissance des difficultés d'analyse et de traitement des problèmes sociaux. Chacun à leur place et à différents moments, ils ont pu constater l'inefficacité relative de politiques sociales souvent trop éclatées et mal coordonnées. La longue quête pour la création du ministère du Travail mais aussi pour l'élargissement du champ de ses compétences est symbolique de ce processus d'hésitations et de tâtonnements (9). Mais le cas français n'est, de ce point de vue, en rien original. Les ministères anglais et américain, bien sûr sur des lignes de partages différentes, restent aussi des institutions en chantier. Les premiers dirigeants du BIT ont conscience de la dynamique internationale de la réforme sociale et de l'insuffisance de ses aboutissements. Ils se sentent portés par la reconnaissance de ces questions mais savent aussi l'urgence de nouvelles initiatives pour faire face aux troubles de l'après-guerre (10).

$\mathrm{Au}$ sein du mouvement ouvrier international, Albert Thomas défend un réformisme s'appuyant sur les quatorze points de Wilson et s'oppose nettement au bolchevisme. Il considère sa nomination comme le signe d'un choix du politique contre une approche plus administrative de la tâche. Le BIT doit formuler une vision internationale de la modernisation économique et sociale. Il faut pour

(9) Concernant la création et l'histoire du ministère du Travail, $c f$. Tournerie (1971), RenARD (2001) et VIET (2001). Sur les étapes concernant la baisse du temps de travail, $c f$. FRIDENSON (2004).

(10) Sur ces développements croisés des deux côtés de l'Atlantique, $c f$. RODGERS (1998). cela constituer et animer une institution nouvelle s'appuyant sur les associations liées par l'exigence internationale de justice sociale reconnue par le traité de paix.

\section{Un domaine de compétences à conquérir}

Le BIT prépare les Conférences internationales du travail et suit la ratification des conventions adoptées lors de ces réunions. Il doit favoriser les échanges internationaux sur les conditions de travail et la vie ouvrière. Son directeur applique une politique définie et contrôlée par le conseil d'administration. Sa marge de manœuvre est limitée. Cependant, le domaine d'action de l'OIT est défini de manière large et abstraite dans les "principes généraux» proposés dans la section II de la constitution. Son préambule énonce «à titre d'exemple» et parmi «d'autres mesures analogues», les «méthodes et principes qui paraissent être d'une importance particulière et urgente» :

«1) le principe dirigeant que le travail ne doit pas être considéré simplement comme une marchandise ou un article de commerce;

2) le droit d'association, en vue de tous les objets non contraires aux lois, aussi bien pour les salariés que pour leurs employeurs;

3) le paiement aux travailleurs d'un salaire leur assurant un niveau de vie convenable tel qu'on le comprend dans leur temps et dans leur pays;

4) l'adoption de la journée de huit heures ou de la semaine de 48 heures comme but à atteindre partout où il n'a pas été obtenu;

5) l'adoption d'un repos hebdomadaire de 24 heures au minimum, qui devrait comprendre le dimanche toutes les fois que ce sera possible;

6) la suppression du travail des enfants et l'obligation d'apporter au travail des jeunes gens des deux sexes les limitations nécessaires pour leur permettre de continuer leur éducation et d'assurer leur développement physique;

7) le principe du salaire égal, sans distinction de sexe, pour un travail de valeur égale;

8) les règles édictées dans chaque pays au sujet des conditions de travail devront assurer un traitement économique équitable à tous les travailleurs résidant légalement dans le pays;

9) chaque État devra organiser un service d'inspection qui comprendra des femmes, afin d'assurer l'application des lois et règlements pour la protection des travailleurs» (11).

Ces principes d'action constituent le socle des attributions de l'OIT mais ne limitent pas strictement sa compétence. Toutes ces questions ne sont pas non plus de son ressort exclusif dans le cadre de la Société des Nations. La question de la santé des travailleurs est aussi du domaine de l'organisation

(11) Traité de Versailles, partie XIII, article 427, BIT, (1931), annexes. 
d'hygiène. La protection des femmes et des enfants concerne également la Commission consultative des questions sociales. Les conditions d'emploi et de rémunération constituent des problèmes économiques et sont partiellement du ressort de l'Organisation économique et financière. Seuls trois domaines sont clairement réservés à l'OIT: la réglementation de la durée du travail, la sécurité sociale et la liberté syndicale. Autour de ce bloc de compétences, sa compétence dépend du partage des attributions avec les organes fonctionnels du système sociétaire, c'est-à-dire consacrés à une tâche spécifique par opposition aux organes politiques. L'OIT est aussi, juridiquement, un organe fonctionnel mais son domaine d'action, le travail, lui confère un rôle et un statut particuliers. L'OIT peut prétendre à une définition large de son champ d'action et à un certain contrôle des activités de ces organisations techniques. La politique de l'OIT dépend d'une négociation permanente avec le secrétariat de la SDN. Dans ce cadre, le rôle du Bureau et de son directeur est fondamental.

Albert Thomas élabore une organisation partagée en trois grandes divisions: la division diplomatique dirigée par l'Irlandais Edward Phelan, la division scientifique ou division des études coordonnée à l'origine par l'Américain Royal Meeker et une division dite politique assurant le contact avec les organisations ouvrières et patronales confiée à l'Italien Di Palma Castiglione. La coordination de leurs actions est assurée par un cabinet réunissant autour d'Albert Thomas et du directeur adjoint, Harold Butler, un service des relations extérieures chargé de la correspondance avec les bureaux à l'étranger, un service des "enquêtes générales» et un service de relations avec la presse. À ce cabinet sont rattachés des services techniques concernant des sujets importants pour le BIT tels que les migrations et le chômage, les assurances sociales ou encore la mécanisation du travail.

Albert Thomas privilégie les contacts directs avec les membres de son administration et s'entoure de collaborateurs souvent choisis parmi les experts ayant travaillé pour les associations internationales ou les ministères concernés. Cette personnalisation relative du pouvoir correspond aux objectifs d'un directeur refusant de limiter son action à celle d'un simple fonctionnaire. Cette structure permet de promouvoir une logique de compétence contre les ingérences nationales et les exigences diplomatiques. Elle rend possible la pérennisation d'un réseau d'influences au sein de nombreuses organisations non gouvernementales. Le BIT cherche ainsi à placer au centre de la vie internationale ses préoccupations sociales et à justifier l'élargissement de ses prérogatives. Dans cette perspective, Albert Thomas s'implique personnellement pour défendre la compétence du BIT en matière d'expertise économique (12).

\section{Une compétence économique contestée}

Albert Thomas lance, dès l'automne 1920, une grande «enquête sur la production». Cette enquête est fondée sur la collecte, à partir de questionnaires envoyés aux organisations patronales des pays membres, de données brutes sur la durée, la productivité, les conditions de travail ou encore les modes de rémunération. Une équipe est constituée autour d'Edgar Milhaud afin d'effectuer une synthèse de ce matériel gigantesque. Mais cette initiative est vite critiquée de toutes parts.

\section{Un développement interne empêché}

En France, la Confédération générale de la production française (CGPF) conseille à ses membres de ne pas répondre. Robert Pinot, représentant du patronat français au conseil d'administration, critique l'interprétation extensive du traité par Albert Thomas. Il accuse le directeur du BIT de vouloir créer un «super-État» et considère cette initiative comme un premier pas vers un contrôle social de l'économie qu'il refuse absolument. Albert Thomas conteste ce procès d'intention(13). Mais, sous la pression du conseil d'administration, la section d'études de la production est finalement dissoute. Edgar Milhaud termine en marge du BIT, et esseulé, la compilation de ces données (14).

Cette querelle de compétences est également l'objet d'un avis de la Cour permanente de justice internationale lors de la contestation par le gouvernement français de la compétence agricole de L'OIT en 1921. En effet, la France soumet à la Cour une question additionnelle concernant «l'examen de propositions tendant à organiser et à développer les moyens de production agricole et l'examen de toutes autres questions de même nature». Albert Thomas vient lui-même plaider à La Haye contre la position française. Le premier avis de la Cour rendue en 1922 affirme le caractère compréhensif du préambule de la partie XIII du traité de paix et donc l'étendue des compétences de l'OIT. Le second avis concernant la question additionnelle, tout en rappelant son incompétence en matière d'organisation des moyens de production, souligne l'impossibilité d'interdire à

(12) Sur l'analyse de ce réseau d'influence concernant la question des migrations internationales, $c f$. RosenTAL (2006).

(13) "Faire du Bureau un super-État? Cette idée certes n'est pas la mienne. C'est vous qui la forgez de toutes pièces, avec toute votre ingéniosité", lettre d'Albert Thomas à Robert Pinot de mai 1921, AN 94 AP 477.

(14) Concernant cette enquête et l'appréhension des problèmes économiques par Albert Thomas, $c f$. Milhaud (1957). 
l'OIT d'examiner les aspects de la question liés aux compétences que lui reconnaît le traité(15).

Aussi, en collaboration, mais aussi en contrepoint de l'activité de l'organisme économique et financier de la SDN, Albert Thomas affirme légitimement la compétence économique du BIT au nom de la recherche indissociable de la "paix économique» et de la «paix sociale»(16). Mais, si le BIT bénéficie d'une forme d'autonomie au sein du système sociétaire, la SDN exerce un contrôle financier sur lui. La quatrième commission de l'Assemblée doit voter son budget. Or, en 1923, la SDN oblige le BIT à renoncer à la quasi-totalité de ses recrutements en réduisant son budget pour 1924 d'un million de Francs Suisses, c'est-à-dire de près de $15 \%$. Cette diminution s'explique certes par la persistance de la crise économique d'après-guerre, mais elle bloque aussi les ambitions d'un directeur trop audacieux. (17) Albert Thomas doit donc chercher des sources alternatives de revenus pour développer une expertise sur des sujets aussi polémiques que l'étude du Scientific management et de la Rationalisation. Il va trouver aux États-Unis les moyens d'analyser ces manières d'organiser le travail.

\section{Une ambition américaine}

Dès sa nomination, Albert Thomas envisage un voyage outre-Atlantique. Le contexte politique et économique du début des années 1920 n'est guère propice à ce projet. L'élection à la présidence de Warren G. Harding sonne le glas des espoirs d'une participation des Etats-Unis à la SDN. Cependant, malgré les réserves du secrétariat de la SDN, il décide de rester près de deux mois en Amérique du Nord à partir de décembre 1922. Albert Thomas a minutieusement préparé une véritable campagne de promotion de l'OIT. Il multiplie les contacts avec les associations américaines, qu'elles soient patronales, ouvrières, politiques, universitaires ou philanthropiques. Il souhaite amorcer une collaboration technique avec des personnalités américaines et préparer l'opinion publique à la possibilité d'une adhésion. Il déplore même publiquement que l'OIT soit toujours, en Amérique, associée à la SDN. Il insiste sur la distinction entre les deux structures et rappelle le précédent allemand.

L'Allemagne et l'Autriche sont, depuis 1919, membres de l'OIT sans être membres de la SDN. Le principe général est celui de l'identité des membres des organisations de la Société des Nations. Tout membre de la SDN fait nécessairement partie de

(15) Pour un exposé des attendus de ces avis, $c f$. FisCHER (1946).

(16) L'Organisme économique et financier de la SDN devient une organisation permanente en 1923, avec, à sa tête, le Britannique Sir Arthur Salter. Sur la création de ces organes techniques, $c f$. GHÉBALI, (1972).

(17) Sur les polémiques concernant le financement de ces institutions internationales, $c f$. Mouton (1986).
l'OIT. Mais l'Assemblée a, de facto, accepté la possibilité du non-respect de l'identité des membres. En effet, alors que la demande d'adhésion des trois États Baltes est rejetée en 1920, elle autorise l'OIT à envisager une admission de ces pays. Cette différenciation des appartenances reste cependant polémique. Elle remet en cause le pouvoir de contrôle et de coordination de la SDN vis-à-vis de ces institutions. La propagande d'Albert Thomas est perçue comme la revendication d'une indépendance que beaucoup considèrent comme illégitime. Ses déclarations font scandale à Genève (18).

Mais le directeur du BIT continue inlassablement ce voyage qu'il considère comme un succès diplomatique. Les dirigeants de l'American Federation of Labor et de la chambre de commerce des États-Unis proposent même d'envoyer une délégation non officielle à la prochaine CIT. Ses espérances sont vite déçues. La chambre de commerce et l'AFL décident finalement de ne pas envoyer d'observateurs. Le gouvernement américain reste très réticent quant à toute coopération officielle. Cependant, Albert Thomas essaye, à partir de ces rencontres, de mieux comprendre les ressorts d'une prospérité insolente. Il cherche un appui pour ses projets à travers l'actualisation de ces contacts transatlantiques si importants pour les milieux réformateurs.

\section{Un lien progressiste d'après-guerre}

Dans cette perspective, de nombreuses réunions sont organisées avec les dirigeants de fondations américaines. Lors de son voyage à Boston, Albert Thomas rencontre ainsi le président du Twentieth Century Fund, Edward A. Filene. Cet entrepreneur est une des grandes figures du mouvement progressiste en Nouvelle-Angleterre. Dirigeant la chaîne de grands magasins Filene's avec son frère Lincoln, il a introduit un système de représentation et de consultation des employés ainsi que des formes de participation aux résultats. En 1912, il a créé la Cooperative League afin d'aider au développement des coopératives de crédit aux États-Unis. En 1922, cette association a été transformée en une fondation appelée Twentieth Century Fund.

Dans la réflexion d'Edward Filene, est centrale la nécessaire combinaison de la modernisation de l'industrie et d'une certaine démocratisation de la société industrielle. Le cercle vertueux créé par l'équilibre entre une production et une consommation de masse est le fondement d'une nouvelle révolution industrielle. Ce nouveau système industriel doit permettre d'organiser une coopération fructueuse entre employeurs et employés. Mais la stabilité à long terme de ce système ne peut être assurée que par son extension internationale. Edward Filene

(18) Sur les problèmes diplomatiques, légaux et budgétaires que pose ce type d'adhésion, $c f$. TORTORA (1980). 
insiste sur la nécessité d'une compensation des variations des cycles économiques américains et européens grâce à l'instauration d'un marché de masse en Europe. Son credo économique l'amène à prôner une coopération étroite des États-Unis aux travaux de la Société des Nations. Au lendemain de la guerre, E.A. Filene participe aux missions américaines en Europe organisées par la chambre de commerce des États-Unis en coordination avec le département du commerce. En avril 1919, il fait partie de la délégation américaine présentant, en marge des négociations du traité de paix, le projet de création de la chambre de commerce internationale. Mais les positions de plus en plus conservatrices de la chambre de commerce des États-Unis et du gouvernement américain l'incitent à envisager d'autres façons de s'investir internationalement (19).

Albert Thomas ne peut qu'être réjoui par cette ferveur internationaliste. Après son expérience lors de la mobilisation industrielle, il perçoit lui aussi les potentialités économique et sociale d'un modèle de production et de consommation de masse. Le directeur du BIT n'aurait certes pas utilisé les mêmes termes, formulé aussi nettement cette primauté donnée à l'organisation économique devant un congrès socialiste ou la CIT. Mais le milliardaire bostonien et l'ancien député socialiste s'accordent à vouloir analyser et influencer le développement international de formes d'«organisation scientifique du travail». Est, ainsi initiée la création d'un organisme lié au BIT et partiellement financé par le Twentieth Century Fund. Cette idée est développée plus avant par un collaborateur d'Albert Thomas, Paul Devinat, et un industriel ami d'E.A. Filene, Henry S. Dennison. Albert Thomas soutient alors activement ce projet d'un "Institut international d'organisation scientifique du travail». En effet, il conçoit sa création comme une ressource supplémentaire pour influencer les débats de la Conférence économique internationale prévue à Genève en 1927 (20).

\section{Une stratégie contrainte de contournement}

Louis Loucheur propose au nom du gouvernement français la tenue d'une Conférence internationale économique en septembre 1925. Dès la fin de ce mois, Albert Thomas, dans un article intitulé «Pour que la Conférence internationale réussisse», insiste sur l'ambition nécessaire à une telle réunion.

(19) Sur l'approche progressiste d'E.A. Filene, $c f$. McQuaid, (2003) et JACOBS (1997).

(20) Sur Albert Thomas et la modernisation économique, $c f$. FINE (1977) et GUÉRIN (1996).

\section{Un programme pour la Conférence économique internationale}

Le directeur du BIT critique les déclarations de Louis Loucheur visant à exclure du champ de compétence de la conférence «les problèmes liés au plan Dawes et les dettes interalliées, les problèmes d'immigration et d'émigration» et de Lord Cecil refusant l'invocation de "la fourniture des matières premières, les relations entre le capital et le travail, l'organisation de la production»(21). Louis Loucheur souhaite, face à la puissance grandissante des États-Unis, favoriser les accords industriels à l'échelle du continent européen. Une organisation économique créée dans le cadre de la SDN pourrait contrôler ce processus et garantir la défense des intérêts des consommateurs (22).

Pour Albert Thomas, cette simple question implique toutes les autres. Il refuse cette séparation des questions économiques et sociales. Le comité préparatoire de la conférence pourrait être l'embryon de ce qu'il appelle un "Conseil économique international ». (23) Il cherche à inscrire cette conférence dans un cadre tripartite. Elle pourrait initier une réforme de la section économique de la SDN et même une modification de la répartition des compétences entre le BIT et cette dernière. Dans ses notes prises au début de la conférence économique, il formule ainsi la réorganisation qu'il souhaiterait à terme établir: "L'initiative de la conférence économique est une chose toute nouvelle qui ne rentre pas, pour ainsi dire primitivement, dans la charte constitutive de la Société des Nations. [...] Un problème nouveau est posé: c'est la place du travail dans la production même. Si on prend l'économie dans son ensemble, notre isolement n'est plus admissible. Au fonds, je reviens à mon idée. La Société des Nations doit être divisée en deux grandes sections: le politique et l'économique mais c'est l'Organisation internationale du travail qui devrait devenir la partie économique» (24).

Cependant, dès la mise en place du comité préparatoire, cette ambition est mise à mal. La note du gouvernement français précisant sa composition ne propose pas une présence officielle du BIT. Il sera seulement consulté lors de la désignation des représentants des intérêts ouvriers et patronaux. Mais Albert Thomas n'abandonne pas tout espoir d'influer sur la conférence. Il essaye de mettre en avant les projets du BIT à travers les contacts qu'il

(21) Titre de l'éditorial, $c f$. Thomas, (1925). Lord Cecil est une des concepteurs britanniques de la SDN. Il est alors responsable au Foreign Office des relations avec les institutions de Genève. (22) Concernant Louis Loucheur et l'organisation internationale de l'économie, $c f$. CARLS (2000).

(23) Ses arguments correspondent à l'idéal de coopération lié à la création du Conseil national économique en juin 1925. Sur le développement de cette institution, $c f$. СНATRIOT (2002).

(24) Notes d'Albert Thomas prises lors de la Conférence économique internationale de mai 1927, archives BIT, CAT 3-1. 
possède dans les réseaux au sein et autour de la Société des Nations. La création de l'IOST revêt dans ce cadre une importance particulière. Cette Conférence économique internationale est ouverte aux experts des pays non-membres de la SDN. Si la question des dettes interalliées et des réparations a été finalement soigneusement laissée de côté, nul ne peut alors ignorer, dans une Europe affaiblie par la guerre, l'importance de la puissance commerciale et financière des États-Unis. Comment envisager la baisse des barrières douanières sans prendre en compte l'importance du tarif américain? Les ententes industrielles envisagées par Louis Loucheur ne sont-elles pas une réponse à l'inquiétante montée en puissance d'une partie des grandes entreprises américaines en Europe? (DE GrAZIA, 2005).

Dans la vision large que revendique Albert Thomas, le Scientific Management (ou la rationalisation) symbolise ce défi américain. La création de l'IOST pérennise les liens établis avec les milieux d'affaires progressistes et les experts américains. Elle vise aussi à mettre au centre des débats économiques le rôle du Bureau. Les analystes contemporains ne s'y trompent pas. Le professeur de droit Georges Scelle caractérise le nouvel Institut comme «le symbole du droit de vue que celles-ci (les études du BIT) possèdent sur le vaste champ des préoccupations d'ordre économique» (SCELle, 1930, p. 92).(25)

\section{Un positionnement d'entre-deux}

Dès le printemps 1926, Albert Thomas prépare l'acceptation par son conseil d'administration de la collaboration avec le Twentieth Century Fund. Il réussit à faire voter par le groupe des travailleurs de l'organe directeur du BIT une résolution soulignant l'importance de l'étude de l'Organisation scientifique du travail. Dans le rapport du directeur présenté à la CIT de juillet 1926, il insiste sur ce point. Albert Thomas inclut dans ce texte des réponses aux critiques de l'action du BIT et envisage ce que devrait être son développement futur. Il fait de ce document une déclaration de politique générale devant la conférence. La discussion des orientations qu'il propose lui permet de jouer de cette légitimité face aux décisions de son conseil d'administration. À la suite de cette déclaration, la conférence demande, par un vote solennel, l'étude de cette question par le BIT.

Edward A. Filene se rend à Genève en septembre 1926 à l'occasion de l'Assemblée de la Société des Nations. Il participe avec enthousiasme à la ferveur internationaliste accompagnant l'admission de l'Allemagne. Lors de cette visite, il met au point un accord définitif sur la création de l'Institut avec Albert Thomas. À cette occasion, le directeur

(25) Cet ouvrage est préfacé par Albert Thomas. du BIT organise une rencontre entre E.A. Filene et Ernest Mercier. Albert Thomas a connu Ernest Mercier lors de la mobilisation industrielle durant la guerre. Ce polytechnicien, magnat de l'électricité fasciné par le développement industriel américain et inquiet de la reconstitution de la puissance allemande, est le principal animateur du redressement Français fondé en décembre 1925. Ce mouvement richement doté par les industriels des secteurs les plus dynamiques de l'industrie française rassemble dans son comité de patronage les dirigeants des grandes associations patronales mais aussi des réformateurs sociaux comme Arthur Fontaine. (26) Suite à son entretien avec Edward Filene, Albert Thomas reçoit Ernest Mercier chez lui. Dès l'immédiat après-guerre, il a œuvré, avec d'autres, au rapprochement des associations internationales de défense des droits sociaux: l'Association internationale pour la protection légale des travailleurs, l'Association pour la lutte contre le chômage et l'Association pour le développement des assurances sociales. Président du premier Congrès les rassemblant à Prague en octobre 1924, il appelle à la fusion des trois structures en une association unique. Un an plus tard, lors d'une session spéciale à Berne le 21 et le 22 septembre 1925, est décidée la création de l'Association internationale pour le progrès social (AIPS). Albert Thomas est alors nommé président de la section française. Albert Thomas assure, dans ce contexte, un lien précieux entre le redressement Français et l'Association internationale pour le progrès social (FINE, 1976).

En ce mois de septembre 1926 où l'AIPS organise également son premier congrès à Montreux, cette rencontre organisée entre Ernest Mercier et E.A. Filene est emblématique de la volonté d'Albert Thomas d'insérer le nouvel institut dans cette nébuleuse modernisatrice où il joue de son influence. Le discours d'Ernest Mercier présenté en mars 1927 durant une séance de l'Association française pour le progrès social concernant «l'aspect social de la rationalisation» trouve sa continuation internationale dans le rapport présenté par Paul Devinat sur «les conséquences sociales de la rationalisation» lors d'une réunion de l'AIPS en septembre 1927. Ernest Mercier présentait les premières conclusions de la section française. Paul Devinat, premier directeur de l'IOST, tente une synthèse de l'enquête de l'AIPS au niveau international(27). Au carrefour entre États-Unis et Europe, entre patrons et ouvriers, avec un pied en dehors et au sein du système de la Société de Nations, Albert Thomas voit le nouvel Institut comme un moyen de se positionner dans cet

(26) Concernant Ernest Mercier et le redressement français, $c f$. KUISEL (1967).

(27) P. Devinat, Les conséquences sociales de la rationalisation économique, rapport général présenté à la deuxième assemblée générale de l'Association internationale pour le progrès social, Vienne, 14-17 septembre 1927, archives BIT, N401. 
entre-deux international qui constitue le lieu privilégié de son action.

\section{Une reconnaissance ambiguë de la « rationalisation»}

Les activités del'IOST commencenten mars 1927. Paul Devinat, son premier directeur, fait alors de la préparation de la Conférence internationale économique l'objectif principal des débuts de l'Institut. La nouvelle institution est invitée par le président de la conférence, le Belge Georges Theunis, à désigner un expert. Son président, l'Italien Francesco Mauro propose un texte au sous-comité de la Commission de l'industrie chargé des problèmes de rationalisation économique. Il est rapidement accepté comme la seule base de rédaction. Après des modifications de détail discutées avec Henri de Peyerimhoff, le rapporteur de la Commission de l'industrie, cette résolution est votée par le sous-comité puis, en séance plénière, par les experts de la conférence. Elle donne une définition large et vague de la «rationalisation ». Elle doit, en effet, viser:

«1) à donner au travail son maximum d'efficacité dans le minimum d'effort;

2) à faciliter par une moindre variété des types - là où celle-ci n'offre pas d'avantages évidents - l'étude, la fabrication, l'emploi et le remplacement des pièces en série;

3) à éviter le gaspillage des matières premières et de l'énergie;

4) à simplifier la distribution des marchandises et;

5) à la dégager des transports illogiques, des charges financières écrasantes et de la superposition inutile des intermédiaires » (28).

Une application adaptée de la «rationalisation» doit assurer aux consommateurs des prix plus bas et des produits plus adaptés à leurs besoins, aux producteurs des salaires plus élevés et plus équitables, et à la communauté dans son ensemble tout simplement une meilleure condition de vie. La conférence reconnaît unanimement la nécessité de la coordination de plus ambitieux efforts dans ce domaine. Elle demande à l'Organisation économique de la Société des Nations d'accompagner l'action des institutions s'occupant spécifiquement de ce sujet et d'en rendre compte dans ses rapports sur les conditions industrielles. La résolution justifie donc la place internationale de l'Institut et son rôle spécifique.

Mais la conférence ne propose pas la création d'une nouvelle structure ou une véritable réforme du comité économique de la SDN. Les séances plénières ont été marquées par l'opposition nette entre deux visions différentes des possibilités de relance de l'économie mondiale. Sir Arthur Salter,

(28) Conférence économique internationale, Genève, mai 1927, archives du BIT, N401. le secrétaire de l'Organisation économique et financière, donne la priorité aux négociations concernant la baisse des barrières douanières et la suppression des entraves au commerce. Louis Loucheur, le représentant de la France au Conseil de la SDN, prône l'instauration progressive d'ententes industrielles internationales par branches. Ces accords impliquant un partage des marchés remettraient en cause la clause de la nation la plus favorisée au profit d'une approche plus régionaliste du développement économique. Ces approches divergentes correspondent notamment au refus de la Grande-Bretagne, mais aussi des États-Unis, de l'esquisse d'une intégration industrielle du continent européen fondée sur des partenariats franco-allemands, à l'instar du cartel international de l'acier(29).

Louis Loucheur et ses partisans n'arrivent pas à imposer leur point de vue. Le sous-comité sur les ententes industrielles se contente d'envisager un suivi et une publicité de ces formes de «coopération industrielle internationale». Pour les promoteurs d'un système d'ententes internationales, la résolution sur la rationalisation devait venir à l'appui de celle sur les ententes afin de définir les principes d'une politique de modernisation économique et son niveau d'action. Au-delà des querelles diplomatiques, ils posaient la question éminemment controversée du rôle des États dans l'organisation des marchés. Or, cette approche est soigneusement laissée de côté.

Le vote de cette résolution est donc un succès ambivalent pour l'Institut. Il n'augure pas de grandes possibilités de coopération avec l'organisation économique de la SDN, mais justifie a posteriori sa création. Il permet une reconnaissance de la légitimité de sa collaboration avec le BIT et de son financement américain. Les résultats de la conférence économique décevants et défavorables pour le BIT en font paradoxalement un outil privilégié pour développer en marge des institutions une expertise unique. L'Institut international d'organisation scientifique du travail devient alors le relais d'expériences originales et une ressource importante pour l'élaboration d'un discours spécifique sur la modernisation du processus de production.

Entre États-Unis et Europe, représentations nationales et revendication d'une approche transnationale, l'Institut réussit, un temps, à incarner la volonté ambiguë d'une rationalisation générale, du niveau le plus local au plus global. En revendiquant l'indétermination d'une réorganisation systémique de la production, il pose, au niveau international, la question épineuse du lien entre Scientific Management et Relations industrielles, entre

(29) Sur ces logiques de coopération régionale, $c f$. BussiÈrE (1993). Sur les initiatives concernant la levée des barrières douanières, $c f$. BADEL (1985). 
modernisation économique et coopération sociale. Son développement permet l'esquisse d'un dialogue original entre justifications managériales et revendications sociales. Il contribue notamment à la mise en perspective des études sur la distribution ainsi que les méthodes de contrôle budgétaire à travers un intérêt pour les formes d'Economic Planning.

Mais cette possibilité de contacts originaux s'efface avec l'aggravation de la crise économique en Europe. L'exaltation des tensions nationalistes et des différences politiques et sociales ne permet pas la continuation de la revendication de cette position modernisatrice d'entre-deux. L'Institut ferme ses portes en janvier 1934 à la suite du refus par le Twentieth Century Fund de continuer son financement. Cette décision est le reflet d'un sentiment de gâchis très profond chez E.A. Filene. Il s'est rendu à la conférence économique de Londres et a suivi les débats conduisant à l'échec retentissant de ce qui, pour lui, avait constitué un objectif majeur, l'organisation d'un apaisement économique en Europe.

Cependant, les thématiques développées par l'Institut sont progressivement intégrées au sein même du BIT par le successeur d'Albert Thomas, Harold B. Butler. Face à la crise et aux difficultés de la SDN, le développement d'une expertise économique est plus que jamais fondamental. H.B. Butler favorise les recherches en matière d'organisation industrielle au sein d'une nouvelle division consacrée spécifiquement aux problèmes économiques. Ses études en partie initiées par l'Institut préfigurent le travail du BIT après la Seconde Guerre mondiale (CAYET, 2005).

Le prisme de ce micro-objet donne à réfléchir sur le rôle persistant des associations internationales privées et l'influence américaine dans les années vingt. L'analyse de la dimension stratégique de l'action du BIT mise en exergue ici impose une déconstruction plus systématique de son fonctionnement institutionnel. Elle souligne la nécessité d'une analyse historique des modes d'institutionnalisation à la marge des circuits internationaux. Dans leur fragilité et leur précarité, ils révèlent à la fois le poids des déterminations et la recherche difficile d'un entre-deux fécond.

Francis Blanchard, ancien directeur du BIT, a intitulé sa contribution aux Mélanges publiés à l'occasion du $75^{\mathrm{e}}$ anniversaire de l'OIT: «Rien que son mandat mais tout son mandat» (BLANCHARD, 1994). Il rappelle dans ce petit texte l'audace historique de sa constitution et de la déclaration de Philadelphie. $\mathrm{Si}$ son mandat lui prescrit de s'exprimer sur les aspects sociaux du développement, l'OIT ne peut laisser de côté les problèmes liés à la politique économique, monétaire et fiscale. Cette limitation est donc bien rhétorique et constitue un appel à une action tous azimuts. Là où le travail se détermine, l'OIT doit être. N'était-ce pas aussi la politique du BIT d'Albert Thomas?

Au-delà des déclarations appelant à une mondialisation juste, définir aujourd'hui une stratégie de long terme pour changer et réarticuler les politiques des organisations internationales est la tâche difficile et fondamentale d'une Organisation internationale du travail en partie marginalisée par le rôle prépondérant des institutions financières de Bretton Woods et de l'Organisation mondiale du commerce nouvellement créée. L'exemple de la création de l'IOST souligne modestement l'importance des organisations non gouvernementales dans la redéfinition de modes d'action à la limite des sphères de compétence. Dans cette perspective, l'analyse du travail acharnée d'Albert Thomas rappelle la multiplicité des initiatives prises alors à la croisée des problèmes économiques et sociaux et à la marge des institutions de la SDN. Il subit nombre d'échecs mais réussit à faire vivre cet espoir d'après-guerre que d'aucuns voulaient oublier. 


\section{Bibliographie}

\section{Fonds d'archives}

Archives du Bureau international du travail à Genève : CAT : cabinet Albert Thomas, N 401 : Organisation scientifique du travail.

Archives nationales françaises, fonds Albert Thomas, 94 AP.

\section{Ouvrages et articles}

BIT (ed.) (1931), Dix ans d'Organisation internationale du travail, Genève, BIT.

BADEL L. (1985), «Trêve douanière, libéralisme et conjoncture (septembre 1929-mars 1930)», Relations internationales, $\mathrm{n}^{\circ}$ 82, été, p. 141-161.

Blanchard F. (1994), «Rien que son mandat mais tout son mandat» in BIT (ed.), Regards sur l'avenir de la justice sociale. Mélanges à l'occasion du 75e anniversaire de l'OIT, Genève, Bureau international du travail.

Bussière E. (1993), «L'Organisation économique de la SDN et la naissance du régionalisme économique en Europe», Relations internationales, $\mathrm{n}^{\mathrm{0}} 75$, automne 1993, p. 301-313.

CARLs S.D. (2000), Louis Loucheur $1872-1931$ : ingénieur, homme d'État, modernisateur de la France, Villeneuved'Ascq, Presses universitaires du Septentrion.

CAYET T. (2005), Organiser le travail, organiser le monde. Étude d'un milieu international d'organisateurs-rationalisateurs durant l'entre-deux-guerres, thèse de doctorat en histoire soutenue à l'Institut universitaire européen de Florence.

Charle C. (1994), «Avant-garde intellectuelle et avantgarde politique: les normaliens et le socialisme (18671914)» in Madeleine Rebérioux et Gilles CANDAR (eds.), Jaurès et les intellectuels, Paris, éditions de l'Atelier, p. 133-168.

Снатвіот A. (2002), La démocratie sociale à la française : l'expérience du Conseil national économique, 19241940, Paris, La Découverte.

De Felice F. (1988), Sapere e Politica. L'organizzazione internazionale del lavoro tra le due guerre 1919-1939, Milano, Franco Angeli.

De Grazia V. (2005), Irresistible Empire. American's Advance through 20th Century Europe, Cambridge, MA, The Belknap Press of Harvard University of Press, 2005.

FINE M. (1976), «Un instrument pour la réforme: l'Association française pour le progrès social (19271929)», Le Mouvement social, $\mathrm{n}^{\circ}$ 94, janvier-mars, p. 45-63.

FINE M. (1977) “Albert Thomas, a reformer's vision of modernization, 1914-32", Journal of Contemporary History, Vol. 12, no 3, July, p. 545-565.
FISHER G. (1946), Les rapports entre l'Organisation internationale du travail et la Cour permanente de justice internationale: contribution à l'étude du problème de la séparation des pouvoirs dans le domaine international, Paris, A. Pedone.

Fridenson P. (2004), «La multiplicité des processus de réduction de la durée du travail de 1814 à 1932 : négociations, luttes, textes et pratiques » in Patrick FRIDENSON et Bénédicte Reynaud, La France et le temps du travail, Paris, Odile Jacob.

GhÉBALI V.-Y. (1972), «Aux origines de l'ECOSOC, l'évolution des commissions techniques de la Société des Nations ", Annuaire français de droit international, p. $469-511$.

GheBALi V.-Y. (1987), L'Organisation internationale $d u$ travail, OIT, Genève, Georg.

GuÉRIN D. (1996), Albert Thomas au BIT, 1920-1932. De l'internationalisme à l'Europe, Genève, Institut européen de l'université de Genève, Euryopa.

JACOBS M. (1997), “Constructing a New Political Economy. Philanthropy, Institution-Building and Consumer Capitalism in the Early Twentieth Century", in Ellen Condliffe Lagemann (ed.), Philanthropic Foundations. New Scholarship, New Possibilities, Indianapolis, Indiana University Press.

Kuisel R.F. (1967), Ernest Mercier. French Technocrat, Berkeley, University of California Press, 1967.

LESPINET-MORET I. (2003), «Arthur Fontaine, grand commis de la nation et ambassadeur du travail», Histoire et Sociétés, avril, p. 111-120.

LuCiani J. (sous la direction de), (1992), Histoire de l'Office du travail (1890-1914), Paris, Syros.

McQuAID K. (2003), A response to industrialism : liberal businessmen and the evolving spectrum of capitalist reform, 1886-1960, Washington D.C., Beard Books, $2^{\text {nd }}$ edition.

Milhaud E. (1957), «Albert Thomas et les problèmes économiques» in Un grand citoyen du monde, Albert Thomas vivant, Genève, Société des amis d'Albert Thomas, p. 147-176.

Mouton M.-F. (1986), «Du super-budget au super-État? Une campagne française contre la $\mathrm{SDN} »$ in Enjeux et Puissance: pour une histoire des relations internationales au $\mathrm{XX}^{\mathrm{e}}$ siècle. Mélanges en hommage à Jean-Baptiste Duroselle, Paris, Publications de la Sorbonne.

Prochasson C. (1999), «Entre science et action: le "réseau Albert Thomas" et le socialisme normalien, 1900-1914» in Christian Topalov, ed., Laboratoires du nouveau siècle, la nébuleuse réformatrice et ses réseaux en France, 1880-1914, éditions de l'EHESS, p. 141158. 
RENARD D. (2001), «Un train peut en cacher un autre. La création du ministère du Travail et de la Prévoyance sociale en 1906 », Revue française des affaires sociales, $\mathrm{n}^{\mathrm{o}} 2$, avril-juin.

Rodgers D.T. (1998), Atlantic Crossings. Social politics in a progressive age, Cambridge, MA, Harvard University Press.

Scelle G. (1930), L'Organisation internationale $d u$ travail et le BIT, Paris, Librairie des sciences politiques et sociales.

Schaper B. W. (1960), Albert Thomas, trente ans de réformisme social (thèse de doctorat, Leyde, 1953), Assen, Van Gorcum, 1959. Traduit du néerlandais. Réédition, Paris, Presses universitaires de France.

Shotwell J. T. (ed.) (1934), The Origins of The International Labor Office, New York, NY, Columbia University Press, 2 vols.

Rosental P.-A. (2006), «Géopolitique etÉtat-Providence. Le BIT et la politique mondiale des migrations dans l'entre-deux-guerres », Annales HSS, janvier-février, $n^{\mathrm{o}} 1$, p. 99-134.
Thomas A. (1925), «Pour que la conférence internationale réussisse», L'Europe nouvelle, 25 septembre, p. 2.

Topalov C. (ed.) (1999), Laboratoires du nouveau siècle, la nébuleuse réformatrice et ses réseaux en France, 1880-1914, Paris, éditions de l'EHESS.

TORTORA M. (1980), Institution spécialisée et organisation mondiale: étude des relations de l'OIT avec la SDN et l'ONU (thèse de science politique, Genève), Bruxelles, Bruylant.

TOURNERIE J.-A. (1971), Le ministère du Travail (origines et premiers développements), Paris, Cujas.

VIET V. (2001), «Cent ans de relations sociales: un fil d'Ariane pour une histoire du ministère du Travail», Revue française des affaires sociales, avril-juin, $\mathrm{n}^{\circ} 2$.

Wieviorka A. (sous la direction de) (2004), Justin Godart, un homme dans son siècle (1871-1956), Paris, CNRS éditions. 


\section{NUMÉRO SPÉCIAL}

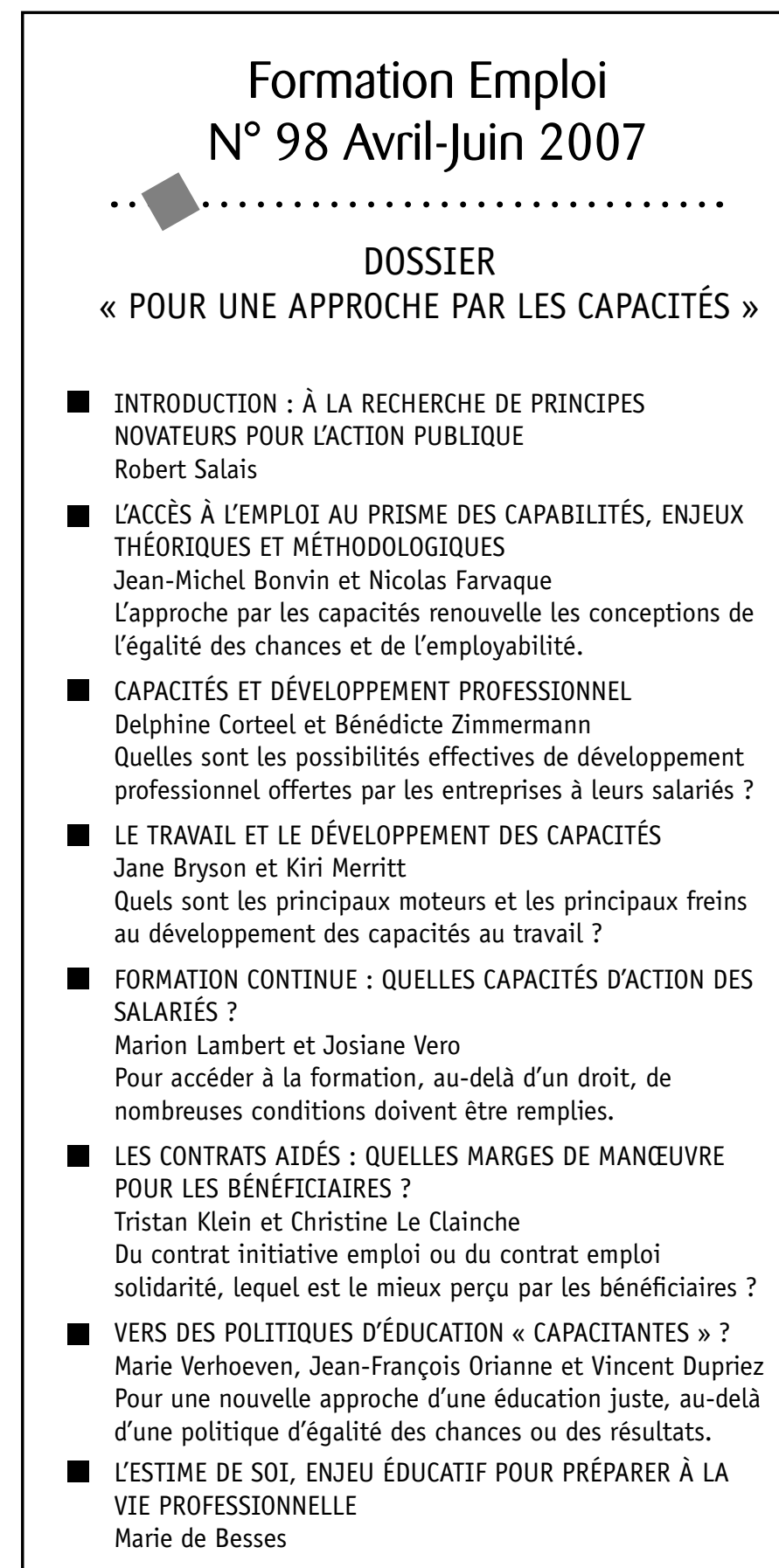

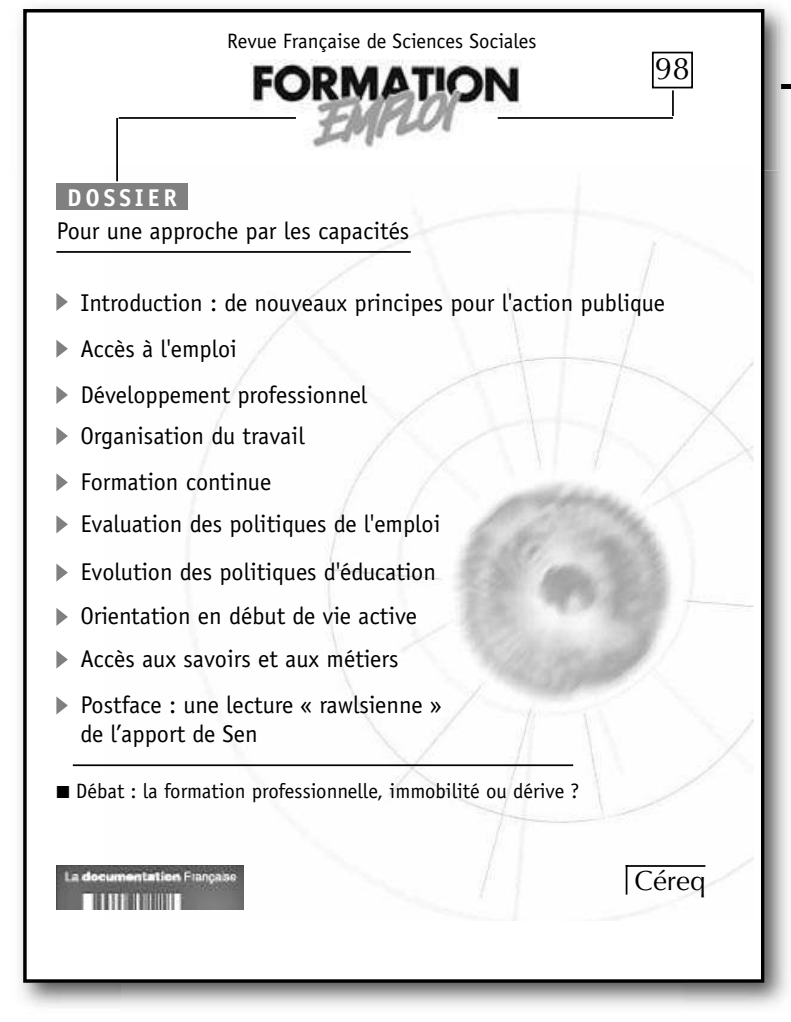

Peut-on aider les jeunes en lycée professionnel à reconsidérer leur situation et leur avenir sous un jour meilleur ?

- QUEL ESPACE DE CHOIX DES MÉTIERS ? LES INÉGALITÉS D'ACCÈS AUX SAVOIRS TECHNIQUES EN GUINÉE MARITIME Jean-Étienne Bidou et Isabelle Droy

L'accès au savoir et aux métiers dépend surtout du cadre familial et du statut social.

- POSTFACE : QUE FAIRE DE « L'APPROCHE PAR LES CAPACITÉS »?

Pour une lecture « rawlsienne » de l'apport de Sen Claude Gamel

\section{DÉBAT}

>> LES DÉRIVES DE LA FORMATION PROFESSIONNELLE Pierre Cahuc et André Zylberberg

>> LA FORMATION PROFESSIONNELLE DES ADULTES : PLUS D'IMMOBILITÉ QUE DE DÉRIVE ?

Pierre Le Douaron

Le numéro: $14,60 € \bullet$ Le numéro spécial : $18,80 € \bullet$ L'abonnement un an (4 numéros) : France $55 €(T \mathrm{TC}) \bullet$ Europe $58,30 €$ (TTC) • Dom/Tom 59,50€(HT) • Autres pays 62,30€(HT) • Supplément avion : 9,05€ • Commande adressée à : La Documentation française 124, rue Henri Barbusse 93308 Aubervilliers Cedex Tél. 0140157000 - Fax. 0140156800 